\title{
Integration of Taste and Calorie Sensing in Drosophila
}

\author{
Jeffrey W. Stafford, Kaylea M. Lynd, Aera Y. Jung, and Michael D. Gordon \\ Department of Zoology, Cell and Developmental Biology, University of British Columbia, Vancouver, British Columbia V6T 1Z3, Canada
}

\begin{abstract}
Animals use gustatory information to assess the suitability of potential food sources and make critical decisions on what to consume. For example, the taste of sugar generally signals a potent dietary source of carbohydrates. However, the intensity of the sensory response to a particular sugar, or "sweetness," is not always a faithful reporter of its nutritional value, and recent evidence suggests that animals can sense the caloric content of food independently of taste. Here, we demonstrate that the vinegar fly Drosophila melanogaster uses both taste and calorie sensing to determine feeding choices, and that the relative contribution of each changes over time. Using the capillary feeder assay, we allowed flies to choose between sources of sugars that varied in their ratio of sweetness to caloric value. We found that flies initially consume sugars according to taste. However, over several hours their preference shifts toward the food source with higher caloric content. This behavioral shift occurs more rapidly following food deprivation and is modulated by cAMP and insulin signaling within neurons. Our results are consistent with the existence of a taste-independent calorie sensor in flies, and suggest that calorie-based reward modifies long-term feeding preferences.
\end{abstract}

\section{Introduction}

An important feature of the neural circuits that control feeding is that they should integrate information on both the palatability (taste) of a food source and its nutritional content. While these variables are often correlated-sugars tend to be a good source of carbohydrates and also have an appetitive sweet taste-there are notable exceptions. For example, some natural and synthetic sweeteners, such as stevioside and saccharin, have an appetitive taste but provide no nutritional value (DuBois and Prakash, 2012). Moreover, sugars with similar caloric content can differ in perceived sweetness by a factor of $>10$ (Biester et al., 1925). In addition to providing a rationale for the utility of tasteindependent calorie sensing, compounds with a disconnect between taste and caloric content provide an excellent opportunity to separate the relative contributions of these two factors to an animal's behavioral response.

Mounting evidence suggests that both mammals and insects have postingestive mechanisms to sense the caloric content of sugars. Trpm5-mutant mice, which are unable to taste sugars, develop a preference for a source of sucrose solution over a source of water (de Araujo et al., 2008). Similarly, the caloric sugar alcohol sorbitol elicits no taste response in flies, yet is capable of

Received April 18, 2012; revised Aug. 21, 2012; accepted Aug. 27, 2012.

Author contributions: M.D.G. designed research; J.W.S., K.M.L., and A.Y.J. performed research; J.W.S., K.M.L., A.Y.J., and M.D.G. analyzed data; J.W.S. and M.D.G. wrote the paper.

We thank Kristin Scott, Douglas Allan, Andrea Manzo, Lisa Dennison, and Peter Cameron for helpful comments on the manuscript. We also thank the Bloomington Stock Center, Kanae lijima-Ando, Vanessa Auld, and Douglas Allan, for generously providing mutant flies, and Alexander Perkins, for advice on the climbing assay. This work was funded by the Canadian Institutes of Health Research (CIHR) Grant MOP-114934. M.D.G. is a CIHR New Investigator. A.Y.J. is the recipient of an Undergraduate Student Research Award from the Natural Sciences and Engineering Research Council of Canada.

The authors declare no competing financial interests.

Correspondence should be addressed to Michael D. Gordon, Department of Zoology, Cell and Developmental Biology, 2350 Health Sciences Mall, University of British Columbia, Vancouver, BC V6T 1Z3, Canada. E-mail: gordon@zoology.ubc.ca.

DOI:10.1523/JNEUROSCI.1887-12.2012

Copyright $\odot 2012$ the authors $\quad 0270-6474 / 12 / 3214767-08 \$ 15.00 / 0$ inducing appetitive memories of associated odors (Burke and Waddell, 2011; Fujita and Tanimura, 2011). Also, a recent study using Drosophila mutants with decreased sugar responses suggests that flies preferentially consume caloric sugar sources independently of taste input (Dus et al., 2011). The molecular and neural mechanisms underlying these taste-independent effects are unknown.

To define the roles taste and nutrition play in flies' feeding decisions when both sensory mechanisms are intact, we developed a behavioral paradigm where flies are presented with a choice between two sugar sources: one with nutritional content and one without. We found that flies initially prefer sugars based on palatability, but over time their preference shifts toward the calorie source. This shift occurs more rapidly in flies that have been fasted, and is modulated by cAMP through its downstream effectors protein kinase $A$ (PKA) and the cAMP response element-binding protein (CREB), as well as by neuronal insulin signaling. Our results support and extend previous studies that have suggested a taste-independent calorie-sensing mechanism in flies and demonstrate that short-term feeding decisions depend largely on palatability, while more long-term preferences are heavily influenced by nutritional content, especially under conditions of food deprivation.

\section{Materials and Methods}

Fly stocks. Flies were reared and maintained on standard cornmeal food at $25^{\circ} \mathrm{C}$ and $70 \%$ relative humidity. Mixed-sex populations of $w^{1118}$ flies were kept for all assays unless noted otherwise. Other fly stocks used were Gr66a-Gal4 (Wang et al., 2004); UAS-shits (Kitamoto, 2001); UAS-InR ${ }^{D N}$ (UAS-InR.K1409A; Bloomington); tsh-gal80 (Clyne and Miesenböck, 2008); UAS-PKIF and UAS-PKIG ${ }^{19,20} F$ (Kiger et al., 1999); UASdCREB2b (Iijima-Ando et al., 2008); UAS-dicer2, nsyb-Gal4, and tubGal80 $^{\text {ts }}$ (McGuire et al., 2004); $d n c^{l}$ (Dudai et al., 1976); Ilp2-Gal4 (Rulifson et al., 2002); and Ilp2, Ilp3, and Ilp5 (Grönke et al., 2010).

Proboscis extension reflex. Proboscis extension reflex (PER) was performed as described previously (Gordon and Scott, 2009). Briefly, flies were food-deprived for $24 \mathrm{~h}$ at $25^{\circ} \mathrm{C}$ in a vial with a wet Kimwipe before 

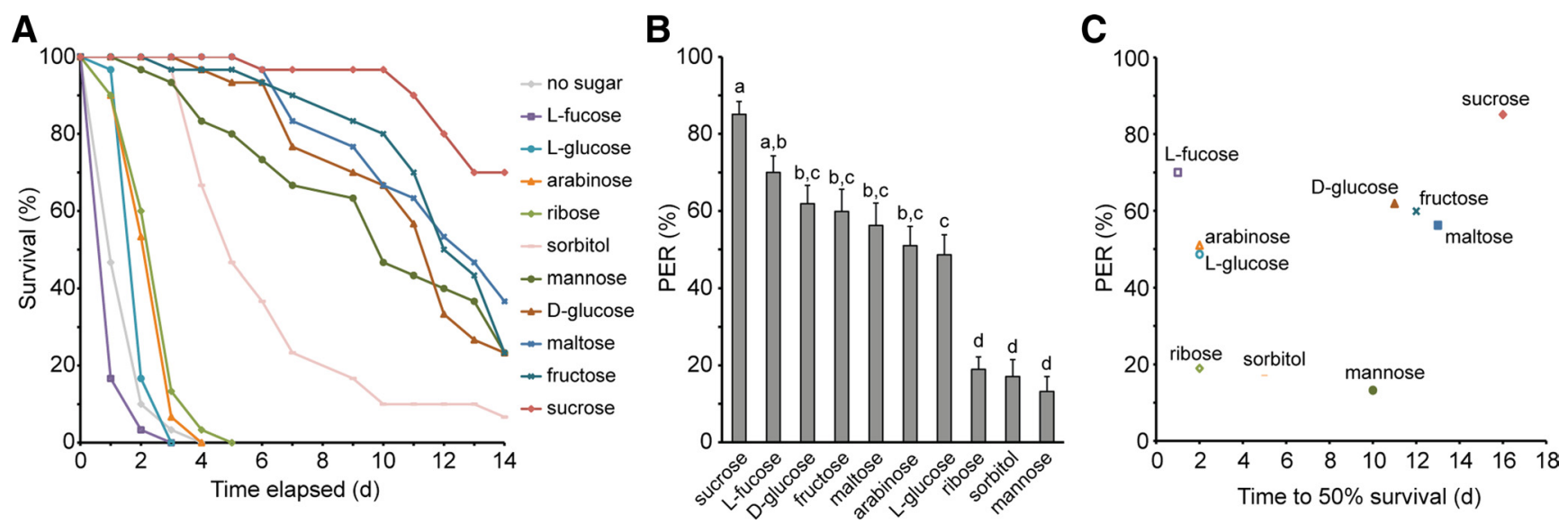

Figure 1. Relationship between sugar palatability and nutritional value. $A$, Survival curves for flies housed on agar plus each indicated sugar at a concentration of $100 \mathrm{~mm} . n=30$ flies for each sugar. $\boldsymbol{B}$, PER of $w^{1118}$ flies stimulated with $100 \mathrm{~mm}$ solutions of each indicated sugar. Data are represented as mean \pm SEM. $n=10$ trials of $8-10$ flies each. Different letters above bars indicate values that are significantly different $(p<0.05)$ by one-way ANOVA with post hoc Tukey's test. C, Plot of PER frequency (a measure of palatability) versus time to $50 \%$ survival (measure of caloric value) for each sugar tested. Note that the survival value of sucrose was estimated based on curve shown in $\boldsymbol{A}$.

the assay. Flies were then immobilized on strips of myristic acid and housed in a humidified chamber before testing. Flies were presented with water and allowed to drink to satiety before each stimulation. PER was measured by touching the tarsi with a droplet of sugar solution and recording the percentage of stimulations that elicit proboscis extension.

Capillary feeder assays. Capillary feeder (CAFE) assays were performed similar to those in previous reports (Ja et al., 2007; Devineni and Heberlein, 2009; Sellier et al., 2011). For all assays, eight female flies were placed in a $15 \mathrm{ml}$ conical vial with four holes in the lid fitted with cut $200 \mu \mathrm{l}$ pipette tips to hold capillaries. The vials had several holes drilled near the base to allow air exchange, and were placed through fitted holes in the lid of a large airtight Tupperware rubber food storage container. The container was filled with a shallow layer of water to ensure high internal humidity. This arrangement allowed air exchange between each vial and the humidified air within the container, thereby minimizing evaporation. Up to 20 vials were placed in the container for each assay. Flies were anesthetized briefly with $\mathrm{CO}_{2}$, placed in each vial, and allowed to recover for $10 \mathrm{~min}$ to $1 \mathrm{~h}$. Two capillaries $(0.5 \mathrm{~mm}$ inner diameter; \#626000, A-M Systems) were filled with each of the sugar solutions being tested and placed through the pipette tips in the lid of each vial. Thus, the flies had access to four capillaries, two containing each of the two solutions. All sugar solutions were composed of the indicated sugars plus $0.01 \%$ FD\&C Blue No. 1 dye for visibility in photographs. Approximately $0.5 \mu \mathrm{l}$ of mineral oil was added to the top of each capillary, and the position of the top meniscus of the sugar solution was marked with a fine marker. The entire apparatus was then photographed with a Pentax Optio W90 handheld digital camera with interval shooting capabilities. One picture was taken each hour for the duration of the assay and ImageJ was used to calculate the volume of solution consumed. Two vials were kept without flies and the disappearance of solution from each of their eight capillaries was averaged and subtracted from the test capillaries to control for evaporation. Preference was calculated according to the following formula: (volume consumed of caloric sugar - volume consumed of noncaloric sugar)/total volume consumed. Preference indices shown reflect the preference within each specified time window, with the exception of Figure $4 \mathrm{~B}$ where cumulative preference (the preference from $t=0$ to each time point) was plotted. All assays were performed in an incubator at $29^{\circ} \mathrm{C}$ with $75 \%$ relative humidity. For experiments using TARGET (temporal and regional gene expression targeting) system, expression was induced by housing the flies at $29^{\circ} \mathrm{C}$ for $5 \mathrm{~d}$ before the assay.

Activity assays. Activity was monitored using the DAM2 system (Trikinetics). Individual flies were placed in tubes with $2 \%$ agar and $5 \%$ sucrose medium. The DAM2 system was kept at $29^{\circ} \mathrm{C}$ to simulate the conditions of the CAFE assays. Activity counts were collected in $10 \mathrm{~min}$ intervals over $16 \mathrm{~h}$, and the average hourly activity was calculated for each fly. At least 10 flies were collected for each genotype in each assay, and each assay was repeated at least twice with similar results.
Geotaxis (climbing) assays. Climbing ability was assessed as described by Perkins et al. (2010) using a modified form of the geotaxis assay (Leal and Neckameyer, 2002). Groups of 10 flies were placed in an empty vial at $29^{\circ} \mathrm{C}$. The flies were briefly tapped to the bottom of the vial and allowed to climb. The number of flies reached a height of $7.5 \mathrm{~cm}$ after $8 \mathrm{~s}$. For each group, the assay was repeated three times and the average value was taken. Climbing index was calculated as the average fraction of successful climbs over at least four independent groups of flies.

Statistics. Details of the statistical tests on each dataset are included in the accompanying figure legends. All statistical tests were performed using GraphPad Prism 5 software.

\section{Results}

\section{Relationship between sugar palatability and} nutritional content

We first wished to define the nutritive content and palatability (appetitive taste) of 10 sugars that previous studies in blowflies and Drosophila (Hassett, 1948; Hassett et al., 1950) suggested to have a range of nutritional and taste qualities: D-sucrose, D-maltose, D-glucose, L-glucose, D-fructose, L-fucose, D-arabinose, D-ribose, D-sorbitol, and D-mannose. To measure nutritional content, we housed adult flies in vials containing $1 \%$ agar with each individual sugar as their sole food source. Consistent with previous results, we found that sucrose, fructose, maltose, D-glucose, mannose, and sorbitol all robustly supported survival, indicating that they provide metabolically accessible energy to the flies (Fig. 1 A) (Hassett, 1948; Hassett et al., 1950; Burke and Waddell, 2011; Fujita and Tanimura, 2011). By contrast, L-fucose, L-glucose, ribose, and arabinose failed to substantially extend lifespan over water controls, suggesting that they have little or no nutritional value.

To measure sugar palatability, we used PER, an established feeding behavior that reports the level of acceptance to taste stimuli (Dethier, 1976; Gordon and Scott, 2009). We stimulated the tarsi of flies with $100 \mathrm{~mm}$ solutions of each sugar and measured the frequency of PER. Largely consistent with previous reports in blowflies, we found that sucrose was the most palatable sugar, followed by L-fucose and D-glucose (Fig. $1 B$ ). By contrast, mannose, ribose, and sorbitol elicited very little PER $(<20 \%)$, indicating that they stimulated little to no response in sugar sensory neurons. Fructose, maltose, L-glucose, and arabinose all elicited intermediate levels of PER (40-60\%; Fig. $1 B$ ).

Plotting the time to $50 \%$ survival versus PER frequency for the sugars tested showed that there is a moderate positive correlation 
A
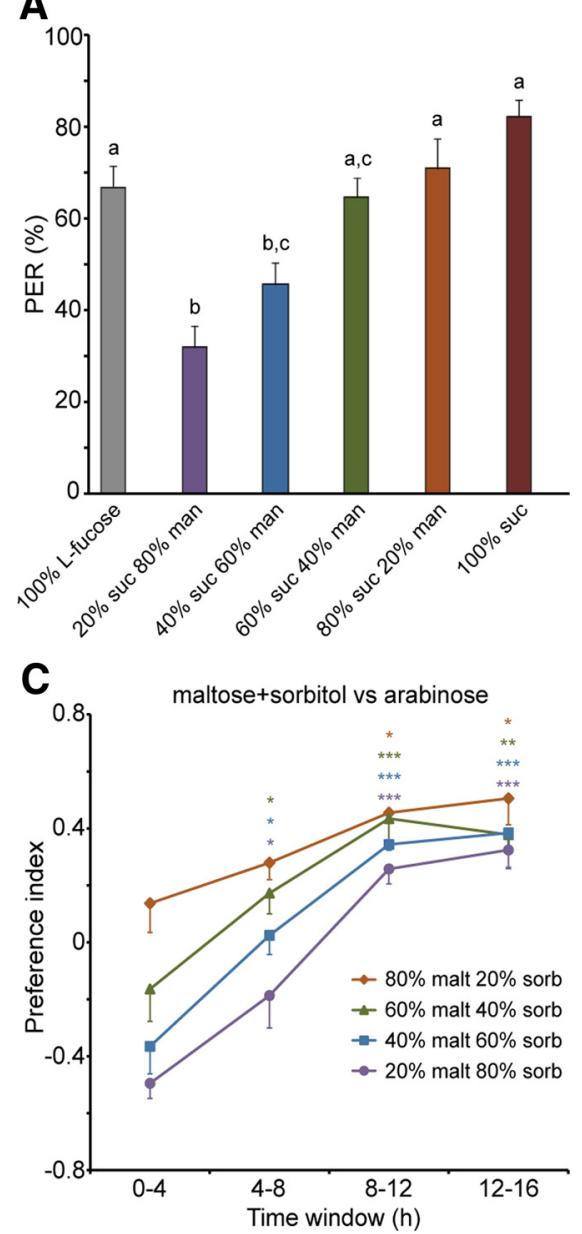

B

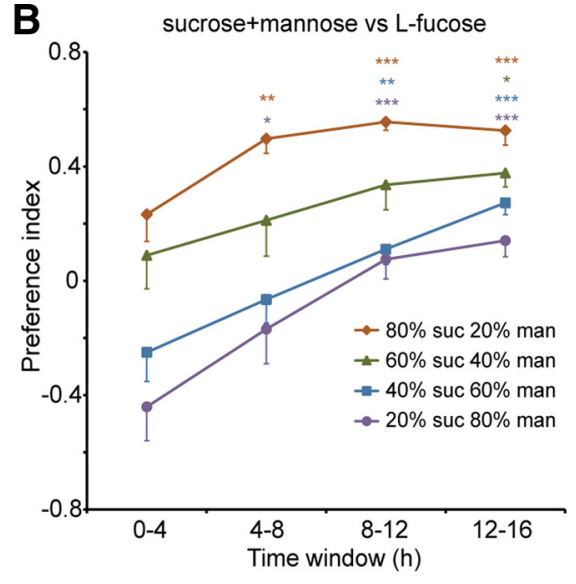

D

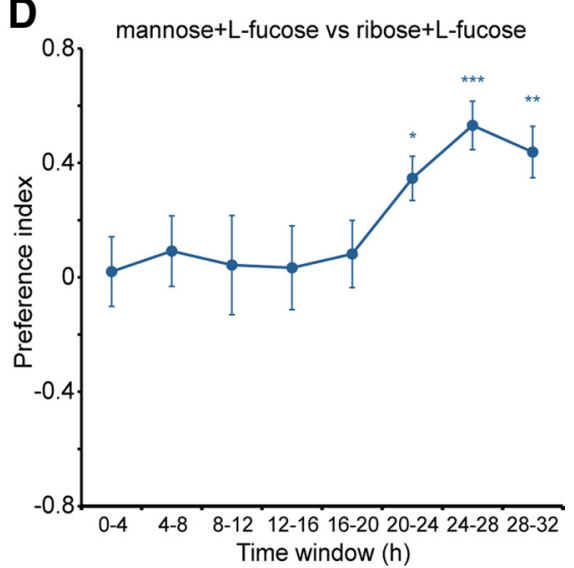

Figure 2. Flies modify sugar preference toward caloric choice over time. A, PER of $w^{1118}$ flies stimulated with $100 \mathrm{~mm} \mathrm{L-fucose}$ or mixtures of sucrose and mannose (always to $100 \mathrm{~mm}$ total concentration). Data are mean \pm SEM. $n=6-8$ trials of $8-10$ flies each. Different letters above bars indicate values that are significantly different $(p<0.05)$ by one-way ANOVA with post hoc Tukey's test. $\boldsymbol{B}-\boldsymbol{D}$, Preference indices of $w^{1118}$ flies given the choice between the following mixtures of caloric and noncaloric sugars: ( $\boldsymbol{B}$ ) sucrose and mannose mixed at varying proportions versus $\mathrm{L}-\mathrm{fucose}, n=12-14 ;(\boldsymbol{C})$ mixtures of maltose and sorbitol versus arabinose, $n=6 ;(\boldsymbol{D})$ a mixture of $80 \%$ mannose and $20 \%$ L-fucose versus a mixture of $80 \%$ ribose and $20 \%$ L-fucose, $n=$ 30. Preferences are calculated in $4 \mathrm{~h}$ time bins. Total concentration of all solutions is $50 \mathrm{~mm}$ in $\boldsymbol{B}$ and $\boldsymbol{C}$ and $250 \mathrm{~mm}$ in $\boldsymbol{D}$. In these and all subsequent preference plots, positive values indicate preference for the caloric option (e.g., sucrose plus mannose) and negative values for the noncaloric option (e.g., l-fucose). Data for these and all subsequent preference graphs are represented as mean \pm SEM. In this figure, color-coded asterisks indicate a preference significantly different from the first time point by one-way repeatedmeasures ANOVA with post hoc Tukey's test, where ${ }^{*} p<0.05,{ }^{* *} p<0.01,{ }^{* * *} p<0.001$. Overall, the significance of the time effect in these experiments was $p<0.0001$.

between nutritional content and palatability (Fig. $1 C ; r^{2}=0.37$ ); however, this is not necessarily reflective of all sugars, as we were biased in our selection to include sugars with a range of nutritional values and levels of palatability. Notable outliers include L-fucose, which is highly palatable but offers little to no nutritional value, and mannose, which elicited the lowest PER response but has high nutritional content.

\section{Flies shift feeding preference toward caloric sugars}

If flies were able to use caloric content to guide their feeding choices, we would expect them to preferentially consume highcalorie sugars over those without nutritional value. To test this, we first mixed sucrose (nutritive and sweet) and mannose (nutritive but not sweet) at various ratios and tested their palatability by PER compared with noncaloric L-fucose. As expected, PER frequency was directly related to the proportion of sucrose in the mixture (Fig. $2 \mathrm{~A}$ ). Moreover, we determined that a mixture of
$60 \%$ sucrose and $40 \%$ mannose elicited a taste acceptance response virtually indistinguishable from that of $\mathrm{L}$-fucose.

PER is an effective measure of palatability; however, because flies do not consume any food during this assay, it cannot reflect any potential postingestive effects of nutritional content. To measure feeding preferences over an extended time period, we presented flies with a choice of $60 \%$ sucrose and $40 \%$ mannose versus $100 \% \mathrm{~L}$-fucose using a modified version of the CAFE assay (Ja et al., 2007; Devineni and Heberlein, 2009; Sellier et al., 2011). Importantly, both choices were presented at the same total concentration $(50 \mathrm{~mm})$ to control for any effects of osmolality. Consumption of each food source during the initial $4 \mathrm{~h}$ of feeding was close to equal, in line with their relative palatability (Fig. $2 B)$. However, over time the flies' preference shifted toward the more nutritional sucrose/mannose mixture. This result held true for other ratios tested; in each case, the initial preference measured over the first $4 \mathrm{~h}$ reflected the relative palatability of each solution, and the preference at later time points shifted toward the caloric option (Fig. 2B). Remarkably, even the $20 \%$ sucrose $/ 80 \%$ mannose solution, which resulted in a strong initial preference for the noncaloric L-fucose alternative, was ultimately preferred by flies by the $12-16 \mathrm{~h}$ time window. These data are consistent with a model in which flies' initial sugar choices are determined largely by taste, but over a matter of hours these choices begin to integrate a measure of caloric content.

To ensure that the observed phenomenon was not specific to the particular sugars tested, we repeated the experiment by comparing the preference of flies for caloric mixtures of maltose and sorbitol versus noncaloric arabinose. Once again, flies' initial preference reflected the relative palatability of the mixture, with higher proportions of maltose resulting in higher preferences for the caloric mixture (Fig. 2C). Similar to our results with sucrose, mannose, and L-fucose, we observed a shift in preference toward the nutritive maltose/sorbitol mixture as the assay progressed over time (Fig. 2C).

Our data are consistent with the idea that calorie-sensing drives change in preference over time; however, one alternative explanation for the observed shift in preference is that the taste sensory response to the noncaloric sugars ( $\mathrm{L}$-fucose and arabinose) decreases more rapidly than the response to sucrose or maltose. To test this idea, we measured the preference of flies for a mixture of L-fucose and mannose versus a mixture of $\mathrm{L}$-fucose and ribose (Fig. 2D). In this experiment, the source of sweetness was the same for each food source, although the L-fucose/mannose mixture is caloric, whereas the L-fucose/ribose mixture is not. As predicted, flies initially showed almost equal preference 
for the two sugars and at later time points the flies shifted their preference toward the nutritive L-fucose/mannose mixture (Fig. 2D). Notably, the preference shift occurred more slowly than in previous experiments, which could reflect a decreased sensitivity of the calorie-sensing mechanism to mannose compared with sucrose or maltose.

The activity of Gr66-expressing "bitter" taste neurons is known to promote behavioral avoidance (Thorne et al., 2004; Wang et al., 2004; Marella et al., 2006). It is conceivable that mannose elicits a behaviorally relevant bitter response that desensitizes over time, thereby leading to a decreased avoidance of this sugar and the observed increased preference for a sucrose/mannose mixture. To rule out this possibility, we tested the involvement of bitter-sensing neurons in the relative preference for sucrose/mannose versus L-fucose. First, as a control we measured the preference of flies for $50 \mathrm{~mm}$ sucrose spiked with $0.15 \mathrm{~mm}$ berberine chloride, which elicits a bitter taste response, versus $50 \mathrm{~mm}$ L-fucose (Fig. 3A). Interestingly, while the addition of berberine to the sucrose caused flies to initially strongly prefer the L-fucose, the aversion to berberine was overcome over time and the flies ultimately preferred the sucrose/ berberine mixture. As predicted, silencing of Gr66a neurons by the expression of $s h i^{t s}$ resulted in a significant increase in the preference for sucrose/berberine (Fig. 3A). Conversely, when we silenced Gr66a neurons and observed the preference of flies for a sucrose/mannose mixture versus L-fucose, we observed no difference from controls (Fig. 3B). Therefore, we conclude that the activity of bitter sensory neurons does not contribute to flies' changing preference for sugars in our assays. Together, our data support a model in which calorie sensing, rather than changes in taste sensitivities, drives the observed shift in preference.

\section{Preference for caloric sugars is potentiated by starvation}

Starvation is known to enhance feeding behaviors and taste sensitivity in flies (Barton Browne, 1975; Scheiner et al., 2004; Farhadian et al., 2012; Hergarden et al., 2012; Inagaki et al., 2012). To test the effects of starvation on preference for caloric sugars, we fooddeprived flies for 8 or $24 \mathrm{~h}$ and measured their preference for the mixture of $20 \%$ sucrose and $80 \%$ mannose versus L-fucose. Food deprivation resulted in a significant increase in the preference for the caloric sucrose/mannose mixture that was most dramatic during the first two $4 \mathrm{~h}$ time windows (Fig. $4 A$ ). We next asked whether the increased preference during the first time window reflected a direct effect of starvation on flies' initial preference, or resulted from an earlier shift in preference toward the caloric sugar source. To answer this question, we examined the preference of flies food-deprived for

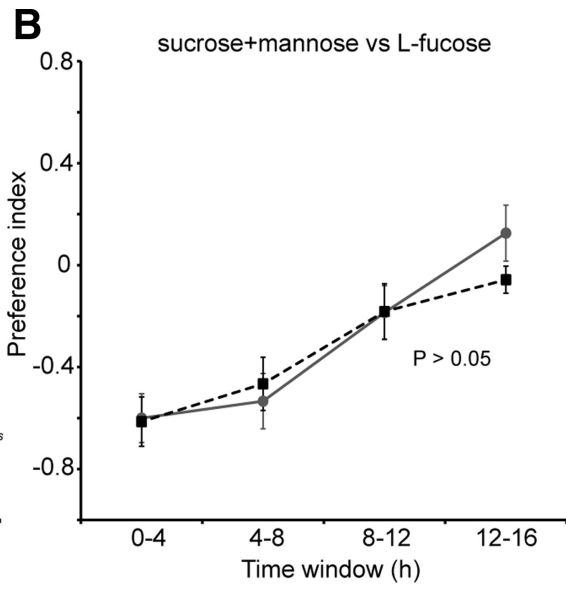

Figure 3. Silencing of bitter neurons affects bitter avoidance but does not affect caloric sugar preference. $\boldsymbol{A}, \boldsymbol{B}$, Preference indices of Gr66a-Gal4/UAS-shits and control flies given the choice between the following: $(\boldsymbol{A})$ a mixture of sucrose and $0.15 \mathrm{~mm}$ berberine chloride versus L-fucose, $n=8 ;(\boldsymbol{B})$ a mixture of $20 \%$ sucrose and $80 \%$ mannose versus L-fucose, $n=8$. Shi ${ }^{\text {ts }}$ expression ollowing figures. Asterisks indicate significant difference between experimental value and controls by two-way repeatedures ANOVA with post hoc Bonferroni test for multiple comparisons, where ${ }^{*} p<0.05,{ }^{* *} p<0.01,{ }^{* *} p<0.001$. Overal
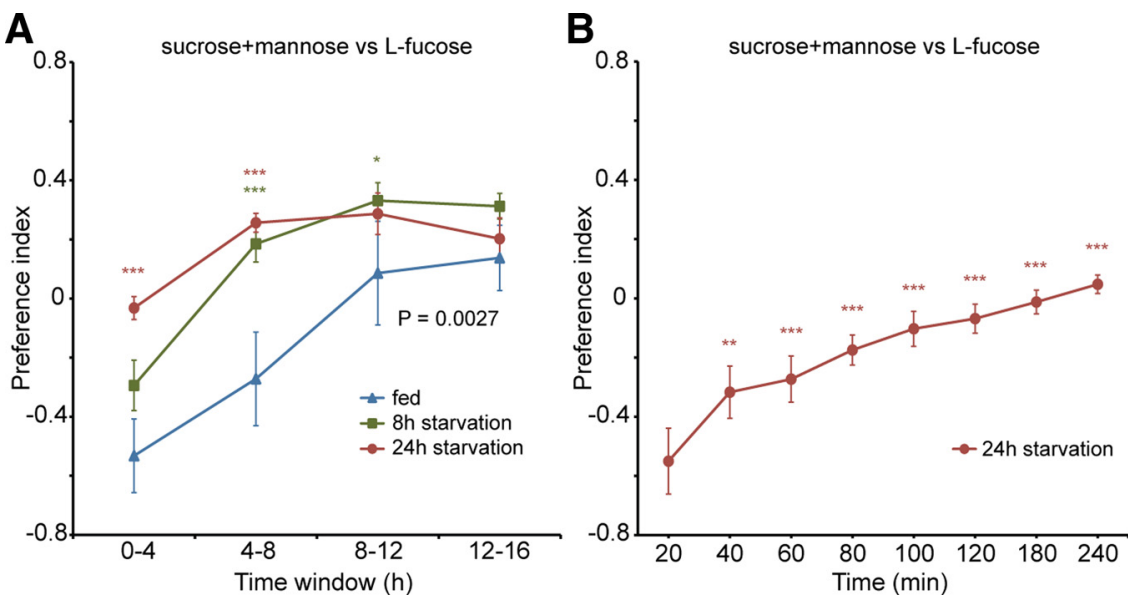

Figure 4. Preference for caloric sugars is potentiated by hunger. $\boldsymbol{A}$, Preference indices of $w^{1118}$ flies given the choice between a mixture of $20 \%$ sucrose and $80 \%$ mannose versus L-fucose, following different lengths of food deprivation. Color-coded asterisks indicate significant difference from fed condition by two-way repeated-measures ANOVA with post hoc Bonferroni test for multiple

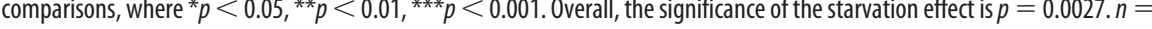
6 trials for each condition. $\boldsymbol{B}$, Cumulative preference over the first $4 \mathrm{~h}$ for flies starved $24 \mathrm{~h}$ in $\boldsymbol{A}$. Asterisks indicate a preference that is significantly different from the first time point by one-way repeated-measures ANOVA with post hoc Tukey's test, where * $p<$ $0.05,{ }^{* *} p<0.01,{ }^{* * *} p<0.001$.

$24 \mathrm{~h}$ in each $20 \mathrm{~min}$ interval during the first $4 \mathrm{~h}$ time window. Our results demonstrate that starved flies have an initial preference very similar to that of fed flies (preference index of - 0.6 ); however, this preference shifts more rapidly in starved flies, resulting in a cumulative preference during the first $4 \mathrm{~h}$ that is close to 0 (Fig. $4 \mathrm{~B}$ ). This starvation-dependent increase in the speed of the preference shift could be due to increased overall consumption (and therefore increased exposure to the caloric option) that results from fooddeprivation and/or an enhancement of the calorie-sensing mechanism under starved conditions.

\section{Sugar preference is modulated by cAMP levels and insulin signaling}

Our observation that preference for caloric sugars develops over time suggests that it may be experience-dependent and involve a 
A
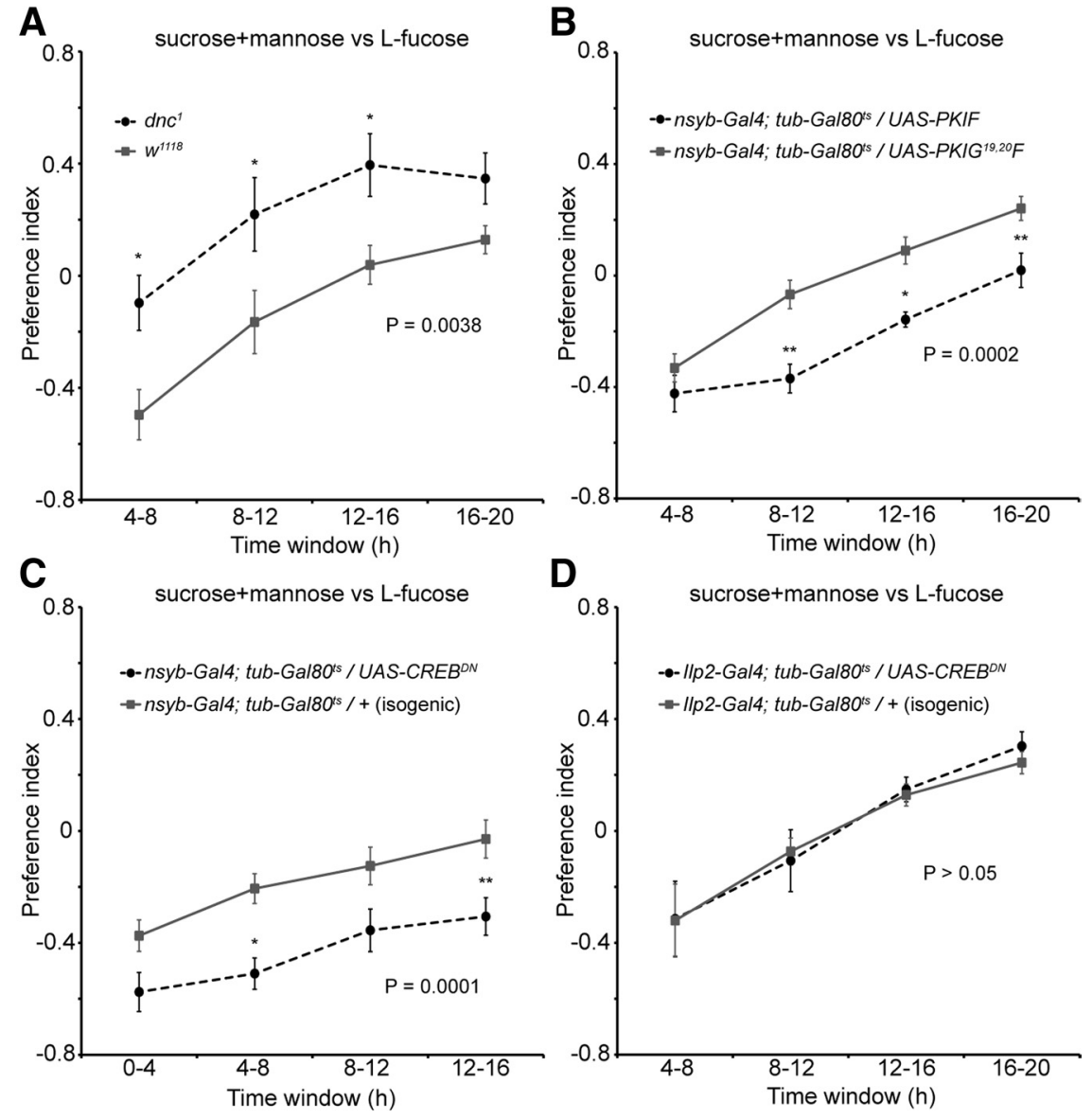

Figure 5. Sugar preference depends on cAMP levels. $\boldsymbol{A}-\boldsymbol{D}$, Preference indices for the following different genotypes given the choice of 20\% sucrose/80\% mannose versus L-fucose: $(\boldsymbol{A}) d n c^{7}$ compared with control $w^{1118}, n=14 ;(\boldsymbol{B})$ flies with pan-neuronal expression of a PKA inhibitor (nsyb-Gal4; tub-Gal80 $/$ UAS-PKIF) compared with control flies expressing an inactive peptide (nsyb-

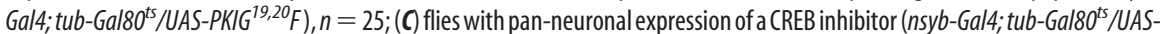
$\left(R E B^{D N}\right.$ ) compared with isogenic control flies (nsyb-Gal4; tub-Gal $\left.80^{t s} /+\right) n=16$; (D) flies expressing CREB ${ }^{\text {DN }}$ in insulin-producing

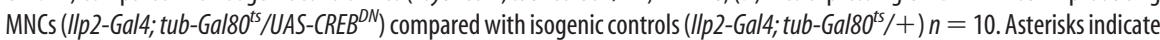
significant difference between experimental value and controls by two-way repeated-measures ANOVA with post hoc Bonferroni test for multiple comparisons, where ${ }^{*} p<0.05,{ }^{* *} p<0.01,{ }^{* * *} p<0.001$. Overall, the genotype effect was significant in $\boldsymbol{A}(p=$ $0.0038), \boldsymbol{B}(p=0.0002)$, and $\boldsymbol{C}(p=0.0001)$, but not in $\boldsymbol{D}(p>0.05)$. In experiments $\boldsymbol{A}, \boldsymbol{B}$, and $\boldsymbol{D}$ and in Figure $6 C, 4-8 \mathrm{~h}$ is shown as the first time window because the flies consumed too little food in the first $4 \mathrm{~h}$ to accurately measure preference.

learning component. Therefore, we wondered whether the process involves pathways known to affect learning and memory. The dunce $(d n c)$ gene encodes a cAMP phosphodiesterase. Mutations in $d n c$ lead to an elevated level of cAMP, which impairs associative learning (Davis and Kiger, 1981). Strikingly, the preference of $d n c$ mutants for a sucrose/mannose mixture over L-fucose was significantly increased compared with controls (Fig. $5 A$ ). This suggests that cAMP signaling may potentiate caloriesensing activity or the downstream behavioral feeding circuits. To further probe the role of cAMP signaling in sugar preference, we targeted the primary effector of cAMP, PKA, within neurons by driving pan-neuronal expression of the PKA inhibitory peptide PKIF or the inactive mutant peptide PKIG ${ }^{19,20} \mathrm{~F}$ (Kiger et al., 1999). In each case, expression was temporally restricted to the adult using the TARGET system to avoid developmental effects (McGuire et al., 2004). Consistent with the elevated preference for nutritive sugars seen in $d n c$ mutants, inhibiting PKA led to a decreased preference for nutritive sugars compared with controls (Fig. 5B). Finally, we inhibited neuronal CREB activity through pan-neuronal expression of the inhibitory isoform dCREB2b $\left(\mathrm{CREB}^{\mathrm{DN}}\right)$ (Iijima-Ando et al., 2008). Similar to the effect of inhibiting PKA, blocking CREB led to a significant decrease in preference for nutritive sugars compared with controls (Fig. 5C). To ensure that the decreased preferences we see after inhibiting PKA or CREB represent a specific feeding defect and are not secondary to an overall decrease in activity or impaired locomotion, we measured activity over a similar $16 \mathrm{~h}$ period and tested the mutants' climbing ability in a geotaxis assay (Fig. 6D). In each case there was no significant behavioral difference compared with controls. Together, our data indicate that neuronal cAMP signaling plays a role in the plasticity mechanism underlying flies' behavioral shift toward caloric sugars.

Since cAMP pathways have been shown to interact with insulin signaling in flies (Wang et al., 2008; Walkiewicz and Stern, 2009) and insulin is regulated in part by hunger state (Ikeya et al., 2002), we wondered whether insulin signaling is also involved in setting flies' preference for caloric sugars. There are seven insulinlike peptides (Ilps) in flies. Ilp2, Ilp3, and Ilp5 are coexpressed in the adult median neurosecretory cells (MNCs), with Ilp3 and Ilp5 expression downregulated in response to starvation, making them good candidates to regulate feeding behavior (Ikeya et al., 2002; Broughton et al., 2005). We tested the preference of Ilp2, Ilp3, and Ilp5 mutants for $20 \%$ sucrose $/ 80 \%$ mannose versus L-fucose. Ilp2 and Ilp3 showed significantly elevated preference for the caloric sucrose/mannose mixture, while Ilp5 showed preference that was not significantly different from that of controls (Fig. 6A). This suggests that multiple Ilps contribute to sugar preference in Drosophila. However, while this could be a direct effect of insulin on feeding circuits, it could also be a secondary effect from insulin's role in metabolism or development. To distinguish between these possibilities, we blocked insulin signaling specifically in the adult fly nervous system. All seven fly Ilps appear to signal through a single, ubiquitously expressed receptor (Fernandez et al., 1995). Neuronal insulin signaling was inhibited through pan-neuronal expression of a dominant-negative form of the insulin receptor $\left(\operatorname{InR}{ }^{D N}\right)$, once again using the TARGET system to restrict the effect to adults (McGuire et al., 2004). We measured the preference of these flies for sucrose/mannose versus L-fucose compared with controls for genetic background. Our results demonstrate that blocking insulin signaling in neurons causes a significant increase in preference for the nutritive sugar option (Fig. 6B). In an effort to begin to localize the effects of insulin activity on feeding circuits, we repeated the same experiment with the introduction of tsh-Gal80 to inhibit InR ${ }^{\mathrm{DN}}$ expression in the thoracic ganglion (Clyne and Miesenböck, 2008). Once again, we saw a shift in flies' preference toward nutritive sugars upon $\mathrm{InR}^{\mathrm{DN}}$ expression (Fig. 6C), demonstrating that at least part of insulin's effect is on circuits in the brain or peripheral sensory neurons. 
PKA and CREB are known to inhibit Ilp release from MNCs during larval development (Walkiewicz and Stern, 2009). Therefore, we wondered whether a similar mechanism underlies the effect of cAMP on preference for nutritive sugars, and could account for the opposing effects of cAMP and insulin signaling on this behavior. However, expression of CREB ${ }^{\mathrm{DN}}$ in the MNCs under control of Ilp2-Gal4 had no effect on preference for a sucrose/ mannose mixture versus L-fucose (Fig. $5 D$ ), suggesting that cAMP acts elsewhere in the nervous system to control sugar preference.

\section{Discussion}

The impact of palatability and nutrition on feeding choices

In mammals and flies, sweet taste plays an important role in promoting the ingestion of sugars, which serve as valuable sources of energy (Yarmolinsky et al., 2009). However, if animals are able to sense the caloric content of sugars directly using a taste-independent mechanism, this raises the important question of what the relative roles of taste and calorie sensing are in driving feeding decisions.

We demonstrate that flies, given the choice between consuming caloric and noncaloric sugar sources, initially choose in strict accordance with taste, or palatability; however, over the course of several hours their preference consistently shifts toward the caloric sugar option, making their long-term feeding preferences much more in line with nutritional content. This suggests that the role of taste is in the immediate or short-term evaluation of food suitability, whereas over time taste becomes less important as flies begin to integrate information about the caloric content of foods sensed through postingestive mechanisms. If this is also true in mammals, it could have important implications in how consumption preferences develop that ultimately have a large impact on health and body weight.

The finding that flies adjust feeding preferences over time has precedent from studies measuring the consumption of alcohol. Flies given the choice between consuming food supplemented with ethanol versus control food gradually shift their preference toward the alcoholic choice over several days (Devineni and Heberlein, 2009). Interestingly, this shift is dependent on cAMP signaling (Xu et al., 2012). Since ethanol is thought to interact with reward circuitry in the brain (Kaun et al., 2011; ShohatOphir et al., 2012), it is tempting to speculate that caloric sugar consumption and ethanol consumption activate similar circuits that ultimately impinge on feeding preference. This is a particularly intriguing possibility given that sugar is known to act as a reward stimulus in associative conditioning of both flies and mammals (de Araujo et al., 2008; Krashes and Waddell, 2008).

Our observation that the preference of flies for one sugar

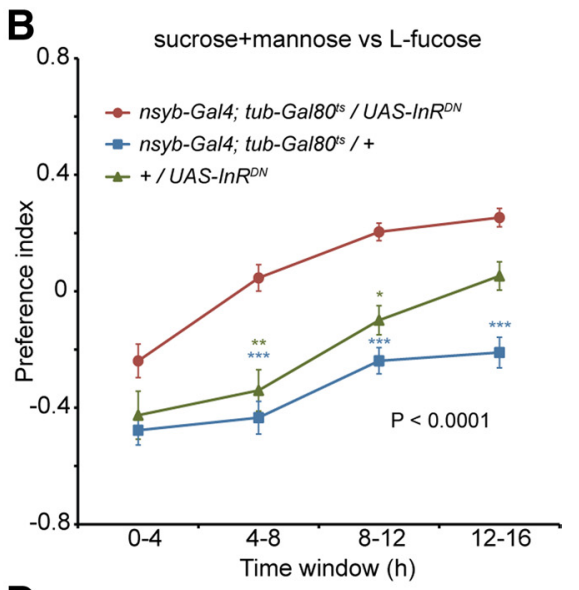

D

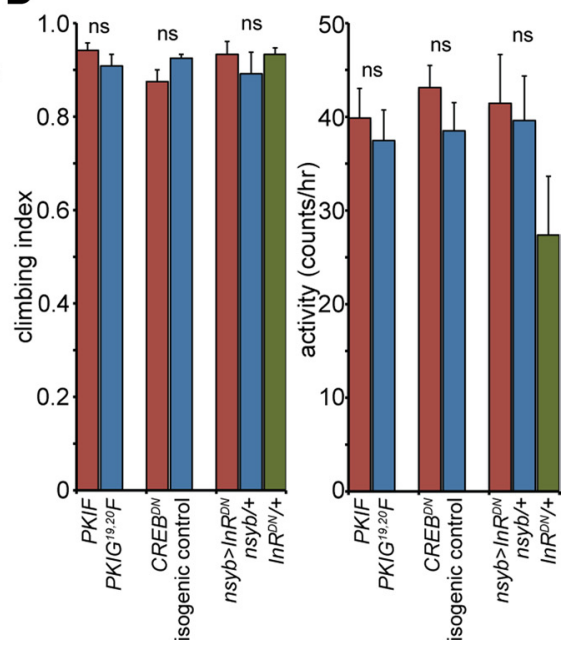

Figure 6. Sugar preference is modulated by insulin signaling. $\boldsymbol{A}-\boldsymbol{C}$, Preference indices for the following different genotypes

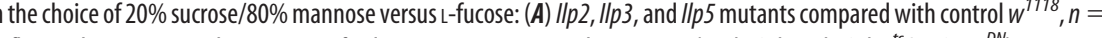
with two control genotypes, $n=30 ;$ (C) flies expressing $\ln R^{\mathrm{DN}}$ in all neurons outside the thoracic ganglia ( $n s y b-G a l 4$, tub-Gal $80^{t s}$ ) UAS-InR $R^{D N}$, tsh-Gal80) compared with two control genotypes, $n=16$. Color-coded asterisks indicate significant difference from $w^{1118}$ in $\boldsymbol{A}$ and flies expressing $\ln \mathrm{R}^{\mathrm{DN}}$ in $\boldsymbol{B}$ and $\boldsymbol{C}$ by two-way repeated-measures ANOVA with post hoc Bonferroni test for multiple comparisons, where ${ }^{*} p<0.05,{ }^{* *} p<0.01,{ }^{* *} p<0.001$. Overall, the genotype effect was significant in both $\boldsymbol{A}$ (all genotypes: indices and activity levels of mutants from $B$ and Figure $5 B, C$. Bars represent mean \pm SEM. Significance between experiment and two controls determined by one-way ANOVA with post hoc Tukey's test. ns, not significant ( $p>0.05$ ).

source over another can drastically change over time also has important experimental implications, as it underscores the importance of considering food preference as a changing metric, rather than a static one. This is especially critical given recent heightened interest in the biological mechanisms underlying feeding control in Drosophila and the number of different implementations of various feeding assays, most of which assess preference only at a single time point (Melcher and Pankratz, 2005; Gordesky-Gold et al., 2008; Moon et al., 2009; Ribeiro and Dickson, 2010; Vargas et al., 2010; Vigne and Frelin, 2010; Dus et al., 2011; Sellier et al., 2011; Weiss et al., 2011).

\section{Flies sense the caloric content of sugars}

Three recent reports suggest that flies have a mechanism to detect the caloric content of food. Two of these studies demonstrated that addition of the nutritious but apparently tasteless sugar alcohol D-sorbitol to noncaloric sugars enhanced the appetitive memory formed for associated odors (Burke and Waddell, 2011; Fujita and Tanimura, 2011). However, these studies did not ad- 
dress the effects of caloric content on dietary choices or whether this was a general phenomenon that extends beyond sorbitol. A more recent study used a genetic approach to closely examine the role of caloric content in directing taste-independent feeding preferences (Dus et al., 2011). It demonstrates that Gr5a, Gr64a double mutants, which have reduced taste sensitivity to a variety of sugars, display a hunger-dependent preference for caloric sugars. Moreover, the authors confirm these results using mutants for the pox-neuro ( poxn) gene, which is required for the development of gustatory bristles. This is an elegant approach, but relies on the potentially troubling assumption that the mutants tested are completely devoid of taste sensory input. While Gr5a, Gr64a mutants show decreased responses to a variety of sugars (Dahanukar et al., 2007), it is not certain that they lack all gustatory sensitivity to the sugars used, especially in taste bristles not easily accessible experimentally, such as those in the mouthparts. Similarly, poxn-null mutants retain a small number of putative chemosensory neurons, most notably in the labral sense organ (Boll and Noll, 2002).

Our results offer important additional support for the existence of a taste-independent calorie-sensing mechanism in flies, as they do not depend on any individual substance being completely tasteless or any mutant being completely taste blind. Instead, we use the relative differences in palatability and nutritional content of various sugars to demonstrate that flies consistently shift their preference toward sugar sources high in available calories. Our study is also the first to control for osmolality, an important taste quality in flies that is sensed by a dedicated population of gustatory neurons known to modulate consumption (Cameron et al., 2010; Chen et al., 2010). Additionally, our approach offers a useful tool in screening for the mechanisms underlying calorie sensing, as it does not depend on any particular mutant background.

An aspect of our conclusions that differs partially from previous studies is the dependence of calorie sensing on starvation. Dus et al. (2011) report that taste-independent preference for caloric sugar sources exists only after $15 \mathrm{~h}$ or more of food deprivation. By contrast, our results suggest that the behavioral shift toward caloric food sources is enhanced by food deprivation, but does not strictly depend on it (Fig. 4). While it is likely that even in our fully fed condition the flies experience partial food deprivation during the assay as they consume the noncaloric sugar, we see significantly increased preference for caloric sugars within the second $4 \mathrm{~h}$ time window, well before they could be attaining a level of hunger nearing that from $15 \mathrm{~h}$ of total food deprivation (Fig. 2). One possible explanation for this discrepancy is that the CAFE assay is a more sensitive measure of feeding than scoring abdomen color following dye consumption. Thus, flies that appear to consume no food based on $2 \mathrm{~h}$ dye ingestion may show quantifiable consumption in the CAFE assay.

\section{Neuronal cAMP and insulin signaling affect sugar preference}

Given cAMP's well established role in neural plasticity, it is perhaps not surprising that it plays an important role in establishing feeding preferences. Indeed, mutants for dnc and rutabaga, an adenylate cyclase, both show decreased feeding discrimination when given a choice between two similar concentrations of sucrose (Motosaka et al., 2007). This is consistent with the observation that each of these mutations interferes with learning and memory. However, our finding that increased cAMP leads to increased preference for caloric sugars, while blocking cAMP signaling decreases preference, suggests a distinct mechanism of action for cAMP in flies' behavioral response to calorie sensing.
The distinct nature of cAMP's role in this behavior compared with established learning paradigms like olfactory conditioning is further supported by our observation that silencing mushroom body output by expression of Shi ${ }^{\text {ts }}$ under control of MB247-Gal4 has no effect on preference for caloric sugars (data not shown).

Insulin signaling controls growth during development and has conserved functions in regulating nutrient storage and metabolism (Britton et al., 2002; Edgar, 2006; DiAngelo and Birnbaum, 2009). Insulin also regulates mammalian feeding by acting as a satiety signal to the brain and inhibiting food reward (Figlewicz and Benoit, 2009). Two of the seven members of the fly insulin gene family, Ilp3 and Ilp5 are transcriptionally downregulated by starvation, making them good candidates to regulate hunger-driven behaviors (Ikeya et al., 2002); however, the exact behavioral effects of insulin signaling in the fly have remained surprisingly obscure. Insulin is known to regulate aversion to bitter compounds in larvae, although this effect could be downstream of metabolic changes in non-neuronal tissues (Wu et al., 2005). Before this study, the only known direct effect of neuronal insulin signaling on adult fly behavior to our knowledge is its role in regulating autocrine short neuropeptide F signaling in sensory neurons of the fly olfactory system to control starvation-induced food-search behavior (Root et al., 2011). Here, we show that blocking neuronal insulin signaling by expression of a dominantnegative insulin receptor leads to a shift in feeding preference toward nutritive sugars. This phenotype parallels the shift in preference seen upon food deprivation, suggesting that downregulation of insulin-like peptides may mediate the hunger-dependent effects on fly calorie sensing.

There are a number of possible mechanisms by which cAMP and insulin signaling may impact feeding circuits. In principle, each could contribute to the neuronal plasticity responsible for shifting feeding preferences toward caloric sugars, or have a direct effect on the calorie sensor itself. Further studies aimed at establishing the molecular mechanisms underlying cAMPdependent and insulin-dependent effects on calorie sensing and pinpointing their specific sites of action will provide important insight into how internal state-dependent peptide signaling modulates behavioral circuits in the fly brain.

\section{References}

Barton Browne L (1975) Regulatory mechanisms in insect feeding. In: Advances in insect physiology (Treherne JE, ed), pp 1-116. London: Academic.

Biester A, Wood MW, Wahlin CS (1925) Carbohydrate studies. I. The relative sweetness of pure sugars. Am J Physiol 73:387-395.

Boll W, Noll M (2002) The Drosophila Pox neuro gene: control of male courtship behavior and fertility as revealed by a complete dissection of all enhancers. Development 129:5667-5681.

Britton JS, Lockwood WK, Li L, Cohen SM, Edgar BA (2002) Drosophila's insulin/PI3-kinase pathway coordinates cellular metabolism with nutritional conditions. Dev Cell 2:239-249.

Broughton SJ, Piper MD, Ikeya T, Bass TM, Jacobson J, Driege Y, Martinez P, Hafen E, Withers DJ, Leevers SJ, Partridge L (2005) Longer lifespan, altered metabolism, and stress resistance in Drosophila from ablation of cells making insulin-like ligands. Proc Natl Acad Sci U S A 102:31053110 .

Burke CJ, Waddell S (2011) Remembering nutrient quality of sugar in Drosophila. Curr Biol 21:746-750.

Cameron P, Hiroi M, Ngai J, Scott K (2010) The molecular basis for water taste in Drosophila. Nature 465:91-95.

Chen Z, Wang Q, Wang Z (2010) The amiloride-sensitive epithelial $\mathrm{Na}^{+}$ channel PPK28 is essential for Drosophila gustatory water reception. J Neurosci 30:6247-6252.

Clyne JD, Miesenböck G (2008) Sex-specific control and tuning of the pattern generator for courtship song in Drosophila. Cell 133:354-363. 
Dahanukar A, Lei YT, Kwon JY, Carlson JR (2007) Two Gr genes underlie sugar reception in Drosophila. Neuron 56:503-516.

Davis RL, Kiger JA Jr (1981) Dunce mutants of Drosophila melanogaster: mutants defective in the cAMP phosphodiesterase enzyme system. J Cell Biol 90:101-107.

de Araujo IE, Oliveira-Maia AJ, Sotnikova TD, Gainetdinov RR, Caron MG, Nicolelis MA, Simon SA (2008) Food reward in the absence of taste receptor signaling. Neuron 57:930-941.

Dethier VG (1976) The hungry fly. Cambridge, MA: Harvard UP.

Devineni AV, Heberlein U (2009) Preferential ethanol consumption in Drosophila models features of addiction. Curr Biol 19:2126-2132.

DiAngelo JR, Birnbaum MJ (2009) Regulation of fat cell mass by insulin in Drosophila melanogaster. Mol Cell Biol 29:6341-6352.

DuBois GE, Prakash I (2012) Non-caloric sweeteners, sweetness modulators, and sweetener enhancers. Annu Rev Food Sci Technol 3:353-380.

Dudai Y, Jan YN, Byers D, Quinn WG, Benzer S (1976) dunce, a mutant of Drosophila deficient in learning. Proc Natl Acad Sci U S A 73:1684-1688.

Dus M, Min S, Keene AC, Lee GY, Suh GS (2011) Taste-independent detection of the caloric content of sugar in Drosophila. Proc Natl Acad Sci U S A 108:11644-11649.

Edgar BA (2006) How flies get their size: genetics meets physiology. Nat Rev Genet 7:907-916.

Farhadian SF, Suárez-Fariñas M, Cho CE, Pellegrino M, Vosshall LB (2012) Post-fasting olfactory, transcriptional, and feeding responses in Drosophila. Physiol Behav 105:544-553.

Fernandez R, Tabarini D, Azpiazu N, Frasch M, Schlessinger J (1995) The Drosophila insulin receptor homolog: a gene essential for embryonic development encodes two receptor isoforms with different signaling potential. EMBO J 14:3373-3384.

Figlewicz DP, Benoit SC (2009) Insulin, leptin, and food reward: update 2008. Am J Physiol Regul Integr Comp Physiol 296:R9-R19.

Fujita M, Tanimura T (2011) Drosophila evaluates and learns the nutritional value of sugars. Curr Biol 21:751-755.

Gordesky-Gold B, Rivers N, Ahmed OM, Breslin PA (2008) Drosophila melanogaster prefers compounds perceived sweet by humans. Chem Senses 33:301-309.

Gordon MD, Scott K (2009) Motor control in a Drosophila taste circuit. Neuron 61:373-384.

Grönke S, Clarke DF, Broughton S, Andrews TD, Partridge L (2010) Molecular evolution and functional characterization of Drosophila insulin-like peptides. PLoS Genet 6:e1000857.

Hassett CC (1948) The utilization of sugars and other substances by Drosophila. Biol Bull 95:114-123.

Hassett CC, Dethier VG, Gans J (1950) A comparison of nutritive values and taste thresholds of carbohydrates for the blowfly. Biol Bull 99:446-453.

Hergarden AC, Tayler TD, Anderson DJ (2012) Allatostatin-A neurons inhibit feeding behavior in adult Drosophila. Proc Natl Acad Sci U S A 109:3967-3972.

Iijima-Ando K, Hearn SA, Granger L, Shenton C, Gatt A, Chiang HC, Hakker I, Zhong Y, Iijima K (2008) Overexpression of neprilysin reduces alzheimer amyloid-beta42 (Abeta42)-induced neuron loss and intraneuronal Abeta42 deposits but causes a reduction in cAMP-responsive elementbinding protein-mediated transcription, age-dependent axon pathology, and premature death in Drosophila. J Biol Chem 283:19066-19076.

Ikeya T, Galic M, Belawat P, Nairz K, Hafen E (2002) Nutrient-dependent expression of insulin-like peptides from neuroendocrine cells in the CNS contributes to growth regulation in Drosophila. Curr Biol 12:1293-1300.

Inagaki HK, Ben-Tabou de-Leon S, Wong AM, Jagadish S, Ishimoto H, Barnea G, Kitamoto T, Axel R, Anderson DJ (2012) Visualizing neuromodulation in vivo: TANGO-mapping of dopamine signaling reveals appetite control of sugar sensing. Cell 148:583-595.

Ja WW, Carvalho GB, Mak EM, de la Rosa NN, Fang AY, Liong JC, Brummel T, Benzer S (2007) Prandiology of Drosophila and the CAFE assay. Proc Natl Acad Sci U S A 104:8253-8256.

Kaun KR, Azanchi R, Maung Z, Hirsh J, Heberlein U (2011) A Drosophila model for alcohol reward. Nat Neurosci 14:612-619.

Kiger JA Jr, Eklund JL, Younger SH, O’Kane CJ (1999) Transgenic inhibitors identify two roles for protein kinase A in Drosophila development. Genetics 152:281-290.

Kitamoto T (2001) Conditional modification of behavior in Drosophila by targeted expression of a temperature-sensitive shibire allele in defined neurons. J Neurobiol 47:81-92.

Krashes MJ, Waddell S (2008) Rapid consolidation to a radish and protein synthesis-dependent long-term memory after single-session appetitive olfactory conditioning in Drosophila. J Neurosci 28:3103-3113.

Leal SM, Neckameyer WS (2002) Pharmacological evidence for GABAergic regulation of specific behaviors in Drosophila melanogaster. J Neurobiol 50:245-261.

Marella S, Fischler W, Kong P, Asgarian S, Rueckert E, Scott K (2006) Imaging taste responses in the fly brain reveals a functional map of taste category and behavior. Neuron 49:285-295.

McGuire SE, Mao Z, Davis RL (2004) Spatiotemporal gene expression targeting with the TARGET and gene-switch systems in Drosophila. Sci STKE 2004:pl6.

Melcher C, Pankratz MJ (2005) Candidate gustatory interneurons modulating feeding behavior in the Drosophila brain. PLoS Biol 3:e305.

Moon SJ, Lee Y, Jiao Y, Montell C (2009) A Drosophila gustatory receptor essential for aversive taste and inhibiting male-to-male courtship. Curr Biol 19:1623-1627.

Motosaka K, Koganezawa M, Narikawa S, Furuyama A, Shinozaki K, Isono K, Shimada I (2007) Cyclic AMP-dependent memory mutants are defective in the food choice behavior of Drosophila. J Comp Physiol A Neuroethol Sens Neural Behav Physiol 193:279-283.

Perkins AD, Ellis SJ, Asghari P, Shamsian A, Moore ED, Tanentzapf G (2010) Integrin-mediated adhesion maintains sarcomeric integrity. Dev Biol 338:15-27.

Ribeiro C, Dickson BJ (2010) Sex peptide receptor and neuronal TOR/S6K signaling modulate nutrient balancing in Drosophila. Curr Biol 20:1000-1005.

Root CM, Ko KI, Jafari A, Wang JW (2011) Presynaptic facilitation by neuropeptide signaling mediates odor-driven food search. Cell 145:133-144.

Rulifson EJ, Kim SK, Nusse R (2002) Ablation of insulin-producing neurons in flies: growth and diabetic phenotypes. Science 296:1118-1120.

Scheiner R, Sokolowski MB, Erber J (2004) Activity of cGMP-dependent protein kinase (PKG) affects sucrose responsiveness and habituation in Drosophila melanogaster. Learn Mem 11:303-311.

Sellier MJ, Reeb P, Marion-Poll F (2011) Consumption of bitter alkaloids in Drosophila melanogaster in multiple-choice test conditions. Chem Senses 36:323-334.

Shohat-Ophir G, Kaun KR, Azanchi R, Mohammed H, Heberlein U (2012) Sexual deprivation increases ethanol intake in Drosophila. Science 335:1351-1355.

Thorne N, Chromey C, Bray S, Amrein H (2004) Taste perception and coding in Drosophila. Curr Biol 14:1065-1079.

Vargas MA, Luo N, Yamaguchi A, Kapahi P (2010) A role for S6 kinase and serotonin in postmating dietary switch and balance of nutrients in $\mathrm{D}$. melanogaster. Curr Biol 20:1006-1011.

Vigne P, Frelin C (2010) Hypoxia modifies the feeding preferences of Drosophila. Consequences for diet dependent hypoxic survival. BMC Physiol 10:8.

Walkiewicz MA, Stern M (2009) Increased insulin/insulin growth factor signaling advances the onset of metamorphosis in Drosophila. PloS One 4:e5072.

Wang B, Goode J, Best J, Meltzer J, Schilman PE, Chen J, Garza D, Thomas JB, Montminy M (2008) The insulin-regulated CREB coactivator TORC promotes stress resistance in Drosophila. Cell Metab 7:434-444.

Wang Z, Singhvi A, Kong P, Scott K (2004) Taste representations in the Drosophila brain. Cell 117:981-991.

Weiss LA, Dahanukar A, Kwon JY, Banerjee D, Carlson JR (2011) The molecular and cellular basis of bitter taste in Drosophila. Neuron 69:258-272.

Wu Q, Zhao Z, Shen P (2005) Regulation of aversion to noxious food by Drosophila neuropeptide Y- and insulin-like systems. Nat Neurosci 8:1350-1355.

Xu S, Chan T, Shah V, Zhang S, Pletcher SD, Roman G (2012) The propensity for consuming ethanol in Drosophila requires rutabaga adenylyl cyclase expression within mushroom body neurons. Genes Brain Behav 11:727-739.

Yarmolinsky DA, Zuker CS, Ryba NJ (2009) Common sense about taste: from mammals to insects. Cell 139:234-244. 